\title{
Full Field Digital Mammography (FFDM) versus CMOS Technology versus Tomosynthesis (DBT) - Which System Increases the Quality of Intraoperative Imaging?
}

\author{
Digitale Vollfeldmammografie (FFDM) versus CMOS-Technologie versus Tomosynthese (DBT) - \\ Welches System steigert die Qualität der Präparateradiografie?
}

Authors

Affiliations
R. Schulz-Wendtland ${ }^{1}$, G. Dilbat ${ }^{2}$, M. Bani ${ }^{3}$, P. A. Fasching ${ }^{3}$, M. P. Lux ${ }^{3}$, E. Wenkel ${ }^{1}$, S. Schwab ${ }^{4}$, C. R. Loehberg ${ }^{3}$, S. M. Jud ${ }^{3}$, C. Rauh ${ }^{3}$, C. M. Bayer ${ }^{3}$, M. W. Beckmann ${ }^{5}$, M. Uder ${ }^{6}$, M. Meier-Meitinger ${ }^{1}$

The affiliations are listed at the end of the article

Key words
breast
mammographic density
mammography
breast cancer
Schlüsselwörter
Mamma
mammografische Dichte
Mammografie
Mammakarzinom

received 26.3.2012 revised $\quad 10.4 .2012$ accepted $\quad 25.4 .2012$

Bibliography

DOI http://dx.doi.org/

10.1055/s-0032-1314942

Geburtsh Frauenheilk 2012; 72:

532-538 @ Georg Thieme

Verlag KG Stuttgart · New York . ISSN 0016-5751

\section{Correspondence}

Prof. Dr. med.

Rüdiger Schulz-Wendtland

Radiologisches Institut des Universitätsklinikums Erlangen

Gynäkologische Radiologie Universitätsstraße 21-23 91054 Erlangen

ruediger.schulz-wendtland@ uk-erlangen.de

\section{Abstract}

Aim: The aim of this prospective clinical study was to assess whether it would be possible to reduce the rate of re-excisions and improve the quality using CMOS technology or digital breast tomosynthesis (DBT) compared to a conventional FFDM system.

Material and Methods: An invasive breast cancer (BI-RADS 5) was diagnosed in 200 patients in the period from $5 / 2011$ to $1 / 2012$. After histological verification, a breast-conserving therapy was performed with intraoperative imaging. Three different imaging systems were used: 1) Inspiration ${ }^{\mathrm{TM}}$ (Siemens, Erlangen, Germany), amorphous selenium, tungsten source, focus $0.1 \mathrm{~mm}$, resolution $85 \mu \mathrm{m}$ pixel pitch, $8 \mathrm{l} / \mathrm{mm}$ as the standard; 2) BioVision $^{\mathrm{TM}}$ (Bioptics, Tucson, USA), flat panel photodiode array, tungsten source, focus 0.05 , resolution $50 \mu \mathrm{m}$ pixel pitch, $12 \mathrm{l} / \mathrm{mm}$; 3) Tomosynthesis (Siemens, Erlangen, Germany), amorphous selenium, tungsten source, focus $0.1 \mathrm{~mm}$, resolution $85 \mu \mathrm{m}$ pixel pitch, $81 / \mathrm{mm}$, range: $50^{\circ}, 25$ projections, scan time $>20$ s, geometry: uniform scanning, reconstruction: filtered back projection. The 600 radiograms were prospectively shown to 3 radiologists.

Results: Out of a total of 200 patients with histologically confirmed breast cancer (BI-RADS 6) 156 patients required no further operative therapy (re-excision) after breast-conserving therapy. A retrospective analysis $(n=44)$ showed an increase in sensitivity with tomosynthesis compared to the BioVision ${ }^{\mathrm{TM}}$ (CMOS technology) and the Inspiration $^{\mathrm{TM}}$ at a magnification of $1.0: 1.0$ of $8 \%$ $(p<0.05)$, i.e. re-excision would not have been necessary in 16 patients with tomosynthesis.

Conclusions: The sensitivity of tomosynthesis for intraoperative radiography is significantly $(\mathrm{p}<0.05)$ higher compared to both CMOS technology and an FFDM system with a conventional detector. Additional studies using higher magnifica-

\section{Zusammenfassung \\ $\nabla$}

Ziel: Prospektive Untersuchung, ob mithilfe der CMOS-Technologie bzw. digitalen Brusttomosynthese (DBT) im Vergleich mit einem herkömmlichen FFDM-System eine Senkung der Rate an Re-Exzisionen und damit eine Qualitätssteigerung möglich ist.

Material und Methode: In der Zeit von 5/2011 bis 1/2012 wurde bei 200 Patientinnen ein invasives Mammakarzinom (BI-RADS 5) diagnostiziert. Nach histologischer Sicherung erfolgte die brusterhaltende Therapie mit intraoperativem Präparateradiogramm mit 3 unterschiedlichen Systemen: 1. Inspiration ${ }^{\mathrm{TM}}$ (Siemens, Erlangen, Deutschland), amorphes Selen, W-Anode, Fokus $0,1 \mathrm{~mm}$, Ortsauflösung $85 \mu \mathrm{m}$ Pixelpitch, $8 \mathrm{l} / \mathrm{mm}$ als Standard. 2. BioVision ${ }^{\mathrm{TM}}$ (Bioptics, Tucson, USA), Photodioden Array Flat Panel, W-Anode, Fokus 0,05, Ortsauflösung $50 \mu \mathrm{m}$ Pixelpitch, $12 \mathrm{l} /$ mm. 3. Tomosynthese (Siemens, Erlangen, Deutschland), amorphes Selen, W-Anode, Fokus $0,1 \mathrm{~mm}$, Ortsauflösung $85 \mu \mathrm{m}$ Pixelpitch, $8 \mathrm{l} / \mathrm{mm}$, Winkelbereich $50^{\circ}, 25$ Projektionen, Scandauer $>20$ s, Geometrie: gleichförmige Abtastung, Rekonstruktion: gefilterte Rückprojektion. Die jeweils 600 Präparateradiogramme wurden prospektiv 3 Radiologen auf einem Befundungsmonitor präsentiert.

Ergebnisse: Bei den 200 Patientinnen mit histologisch gesichertem Mammakarzinom (BI-RADS 6) war bei 156 Patientinnen nach erfolgter brusterhaltender Therapie keine weitere operative Therapie (Re-Exzision) indiziert. Die retrospektive Analyse ( $n=44)$ ergab eine Steigerung der Sensitivität der Tomosynthese sowohl gegenüber des BioVision $^{\mathrm{TM}}$ (CMOS-Technologie) als auch dem Inspiration $^{\mathrm{TM}}$ bei einer Vergrößerung von 1,0:1,0 von $8 \%(\mathrm{p}<0,05)$, d.h. bei 16 Patientinnen wäre mit der Tomosynthese eine Re-Exzision nicht notwendig gewesen. 
tion, e.g. 2.0:1.0, but no zooming will be necessary to evaluate the method further.
Schlussfolgerung: Der Einsatz der Tomosynthese zur Präparateradiografie erreicht eine signifikant $(p<0,05)$ bessere Sensitivität im Vergleich sowohl zur CMOS-Technologie als auch einem FFDM-System mit herkömmlichem Detektor. Studien unter Verwendung höherer Vergrößerungen, z.B. 2,0:1,0, jedoch kein Zooming, sind notwendig zur weiteren Evaluierung der Methode.

\section{Introduction \\ $\nabla$}

A woman's risk of developing breast cancer sometime in her life is around $10 \%$. Breast cancer is thus the most common malignant neoplasm in women and the most common cause of death in women between the ages of 35 and 55 years. In Germany, every year 55000 women develop breast cancer and 20000 die of it. The individual prognosis for women with breast cancer depends, above all, on the extent of the micro-metastases and the general spread of disease at the time of diagnosis and therapy [1-7].

The European Guidelines and the S3 Guidelines require that at least $70 \%$ of all malignant breast lesions (BI-RADS 5) are histologically confirmed preoperatively - the target figure is 90\% [8-11]. The goal of preoperative histological verification of a diagnosed malignant lesion is that only a single operative intervention will be necessary. Preoperative histological examination also permits all non-palpable breast lesions to be marked prior to the actual operative intervention (e.g. wire marking) [8-11].

Radiography is the method of choice to demonstrate complete removal of a lesion. One approach consists of transporting the resected specimen in a suitable container from the operating room directly to the radiology department for imaging [8-11]. In the optimal case, it would be better to carry out imaging ( 2 planes) of the specimen directly in the operating room. The findings could then be communicated directly both to the operating surgeon and to the pathologist, either on film (analogue/digital) or, even more efficiently, via PACS.

The re-excision rate in the literature for breast cancers after primary breast-conserving therapy for previously histologically confirmed breast cancer lesions (histologically B5 and thus BIRADS 6 ) ranges between 10 and 57\%, depending on the defined safety margin $[12,13]$.

The aim of our prospective clinical study was to see whether the use of CMOS technology (Bioptics) and Tomosynthesis (Siemens) could reduce the re-excision rate compared to established digital full field mammography (Inspiration ${ }^{\mathrm{TM}}$, Siemens) in women with histologically confirmed invasive breast cancer undergoing breast-conserving therapy after wire marking of the lesion.

\section{Material and Methods}

$\nabla$

An invasive breast cancer lesion (BI-RADS 5) diagnosed in 200 patients in the period from 5/2011 to $1 / 2012$ using complementary diagnostics (clinic, mammography, sonography, MRT). Patient age ranged from 24 to 87 years (mean: 61 years). Lesion sizes, depending on the imaging method used, were between $4 \mathrm{~mm}$ and $18 \mathrm{~mm}$ (mean: $14 \mathrm{~mm}$ ). All patients underwent targeted punch biopsy or stereotactic vacuum-assisted biopsy preoperatively in accordance with the European Guidelines and the S3 Guidelines [8-11], and all lesions were classified histologically as B5 and diagnostically as BI-RADS 6 . As none of the women had multifocal or multicentric lesions and all tumour diameters were less than

\section{Einleitung}

\section{$\nabla$}

Das Risiko einer Frau, im Laufe ihres Lebens an einem Mammakarzinom zu erkranken, beträgt etwa 10\%. Das Mammakarzinom ist damit die häufigste bösartige Geschwulst der Frau und die häufigste Todesursache für Frauen zwischen dem 35. und 55. Lebensjahr. In Deutschland erkranken pro Jahr 55000 Frauen am Mammakarzinom, 20000 versterben daran. Die individuelle Prognose von Patientinnen mit Mammakarzinom wird vor allem von dem Ausmaß der Mikrometastasierung und Generalisierung der Erkrankung zum Zeitpunkt der Diagnose und der Therapie bestimmt [1-7].

Sowohl gemäß der European Guidelines als auch der S3-Leitlinien wird gefordert, dass mindestens $70 \%$ aller malignen Brustläsionen (BI-RADS 5) präoperativ histologisch zu verifizieren sind - gewünscht werden sogar 90\% [8-11]. Hierdurch soll erreicht werden, dass durch die präoperative Abklärung eines diagnostisch malignen Prozesses in Folge nur ein einzeitiger operativer Eingriff notwendig wird. Hinzukommend sind vor dem eigentlichen operativen Eingriff alle nicht palpablen Brustläsionen entsprechend zu markieren (z. B. Draht) [8-11].

Die Präparateradiografie wird vielfach dann als Mittel der Wahl zum Nachweis der vollständigen Entfernung der Läsion angesehen. Als möglicher Weg wird dabei aufgezeigt, das Präparat vom OP-Saal zur Radiologie in einem entsprechenden Behältnis zu transportieren, um dort direkt geröntgt zu werden [8-11] oder aber optimiert direkt intraoperativ im Operationssaal die Präparateradiografie (2 Ebenen) anzufertigen. Das Ergebnis der Präparateradiografie wird anschließend direkt sowohl dem Operateur als auch dem Pathologen mitgeteilt, entweder durch das Medium Film (analog/digital) oder aber effizienter direkt über das PACS.

Die Mamma-Re-Exzisionsrate nach primär brusterhaltender Therapie (BET) bei zuvor histologisch nachgewiesenem Mammakarzinom (histologisch B5 und damit BI-RADS 6) beträgt in Abhängigkeit des definierten Sicherheitssaums in der Literatur zwischen 10 und $57 \%[12,13]$.

Das Ziel unserer prospektiven Untersuchung war es zu evaluieren, ob mithilfe der CMOS-Technologie (Fa. Bioptics) und der Tomosynthese (Fa. Siemens) im Vergleich mit einem etablierten digitalen Vollfeld-Mammografiegerät (Inspiration ${ }^{\mathrm{TM}}$, Fa. Siemens), eine Senkung der Rate an Re-Exzisionen bei histologisch gesichertem invasivem Mammakarzinom und anschließend folgender brusterhaltender Therapie nach Drahtmarkierung möglich ist.

\section{Material und Methoden \\ $\nabla$}

In der Zeit von 5/2011 bis 1/2012 wurde bei 200 Patientinnen aufgrund der komplementären Diagnostik (Klinik, Mammografie, Sonografie, MRT) ein invasives Mammakarzinom (BI-RADS 5) diagnostiziert. Das Alter der Patientinnen lag zwischen 24 und 87 Jahren (median: 61 Jahre). Die Größe der Läsionen lag nach den 
Table 1 Important basic technical characteristics and features of the systems (system 1: Inspiration ${ }^{\mathrm{TM}}$; system 2: BioVision ${ }^{\mathrm{TM}}$ ) for specimen radiograms. Tomosynthesis (system 3) creates projection slides/cine mode (angular range: $50^{\circ}, 25$ projections, scan time $>20 \mathrm{~s}$, uniform scanning geometry, reconstruction of filtered back projections).

\begin{tabular}{|c|c|c|c|}
\hline & System 1: Inspiration ${ }^{\mathrm{TM}}$ & System 2: BioVision ${ }^{\mathrm{TM}}$ & System 3: Tomosynthesis \\
\hline Manufacturer & Siemens & Bioptics & Siemens \\
\hline Anode & Mo, W & W & Mo, Rh \\
\hline Filter & Mo, Rh & beryllium & Mo, Rh \\
\hline Scattered radiation grid & linear & no & linear \\
\hline Conversion material & $\begin{array}{l}\text { semiconductor } \\
\text { a-Se }\end{array}$ & $\begin{array}{l}\text { photon conversion material: Gd202S: Tb (terbium- } \\
\text { doped gadolinium oxysulfide) phosphorus }\end{array}$ & $\begin{array}{l}\text { semiconductor } \\
\text { a-Se }\end{array}$ \\
\hline Selection process & thin film transistors & progressive line-scan selection & thin film transistors \\
\hline Pixel size & $85 \mu \mathrm{m}$ & $50 \mu \mathrm{m}$ & $85 \mu \mathrm{m}$ \\
\hline Spatial resolution (Nyquist frequency) & $8 \mathrm{Lp} / \mathrm{mm}$ & $12 \mathrm{Lp} / \mathrm{mm}$ & $8 \mathrm{Lp} / \mathrm{mm}$ \\
\hline Field size & $24 \times 30 \mathrm{~cm}^{2}$ & $24 \times 30 \mathrm{~cm}^{2}$ & $24 \times 30 \mathrm{~cm}^{2}$ \\
\hline
\end{tabular}

$2.0 \mathrm{~cm}$, no neoadjuvant chemotherapy was required in accordance with the S3 Guidelines [10,11].

After histological classification as B5 and diagnostic classification as BI-RADS 6, all patients underwent targeted wire marking preoperatively, similar to the primary procedure used for histological verification, using either sonography or stereotactic radiography. This was followed by breast-conserving therapy including the sentinel lymph nodes (marking was carried out using acid blue or technetium $99 \mathrm{~m}$ ). The resected specimen was then marked (for orientation) (3 planes) and radiologically investigated (without compression) around the wire still placed in the segment [14] under AEC (automatic exposure control) using 3 different systems (Inspiration ${ }^{\mathrm{TM}}, 2$ planes, system 1; BioVision ${ }^{\mathrm{TM}}$, 2 planes, system 2; Tomosynthesis, 1 plane, system 3) magnification 1.0:1.0 (๑ Table 1):

1. Inspiration ${ }^{\mathrm{TM}}$ (Siemens, Erlangen, Germany), amorphous selenium, tungsten source, focus $0.1 \mathrm{~mm}$, spatial resolution $85 \mu \mathrm{m}$ pixel pitch, $8 \mathrm{l} / \mathrm{mm}$, the standard.

2. BioVision ${ }^{\mathrm{TM}}$ (Bioptics, Tucson, USA), flat panel photodiode array, tungsten source, focus 0.05 , spatial resolution $50 \mu \mathrm{m}$ pixel pitch, $12 \mathrm{l} / \mathrm{mm}$.

3. Tomosynthesis (Siemens, Erlangen, Germany), amorphous selenium, tungsten source, focus $0.1 \mathrm{~mm}$, spatial resolution $85 \mu \mathrm{m}$ pixel pitch, $8 \mathrm{l} / \mathrm{mm}$, range $50^{\circ}, 25$ projections, scan time $>20$ s, geometry: uniform scanning, reconstruction: filtered back projection.

Radiological images (digital full field mammography: Inspiration $^{\mathrm{TM}}$, 2 planes, standard; CMOS technology, Bioptics, 2 planes; Tomosynthesis, 1 plane, 25 projections, cine mode) of the 200 segments resected during breast-conserving therapy were shown prospectively to 3 radiologists with varying degrees of experience in digital mammography, randomly allocated to the respective radiologist (random generator) who were only aware of the initial findings (histological classification B5, diagnostic classification BI-RADS 6). Images were shown intraoperatively to the radiologists on an monitor ( Figs. 1 to 3 ). The results of the findings (focal lesion with sufficient safety margins $[1.0 \mathrm{~cm}]$ ) for all 3 types of radiological images [2 planes or 25 projections] or not) $[10,11]$ were correlated with the subsequent detailed histological findings; all 3 radiologists came to similar assessments. bildgebenden Verfahren zwischen $4 \mathrm{~mm}$ und $18 \mathrm{~mm}$ (median: $14 \mathrm{~mm}$ ). Gemäß der European Guidelines und der S3-Leitlinien [8-11] erfolgte bei allen Patientinnen präoperativ eine sonografisch gezielte Stanz- bzw. stereotaktisch/röntgenologisch geführte Vakuumbiopsie mit dem histologischen Ergebnis B5, diagnostisch dann BI-RADS 6. Da in allen Fällen keine Multifokalität bzw. -zentrizität bestand und die Größenausdehnung unter $2,0 \mathrm{~cm}$ lag, erfolgte aufgrund der S3-Leitlinien keine primäre neoadjuvante Chemotherapie $[10,11]$.

Nach der histologischen Diagnose B5, diagnostisch BI-RADS 6, wurde bei allen Patientinnen präoperativ entsprechend dem primären Verfahren der histologischen Sicherung der Befunde eine sonografisch geführte oder aber stereotaktisch/röntgenologisch gezielte Drahtmarkierung durchgeführt. Anschließend erfolgte eine brusterhaltende Therapie einschließlich Sentinel Lymph Node (Markierung sowohl mit Patentblau als auch mit Technetium-99m). Das operierte Segment wurde daraufhin fadenmarkiert (Orientierung) (3 Ebenen) und bei im Segment noch platzierten Draht radiologisch kompressionsfrei [14] mit 3 unterschiedlichen Systemen und jeweils unter AEC (Automatic Exposure Control) untersucht (Präparateradiografie) (Inspiration ${ }^{\mathrm{TM}}, 2$ Ebenen, System 1; BioVision ${ }^{\mathrm{TM}}, 2$ Ebenen, System 2; Tomosynthese, 1 Ebene, System 3) jeweils Vergrößerung 1,0:1,0 (๑ Tab. 1):

1. Inspiration ${ }^{\mathrm{TM}}$ (Siemens, Erlangen, Deutschland), amorphes Selen, W-Anode, Fokus 0,1 mm, Ortsauflösung $85 \mu \mathrm{m}$ Pixelpitch, $8 \mathrm{l} / \mathrm{mm}$ als Standard.

2. BioVision ${ }^{\mathrm{TM}}$ (Bioptics, Tucson, USA), Photodioden Array Flat Panel, W-Anode, Fokus 0,05, Ortsauflösung $50 \mu \mathrm{m}$ Pixelpitch, $12 \mathrm{l} / \mathrm{mm}$.

3. Tomosynthese (Siemens, Erlangen, Deutschland), amorphes Selen, W-Anode, Fokus 0,1 mm, Ortsauflösung $85 \mu \mathrm{m}$ Pixelpitch, $81 / \mathrm{mm}$, Winkelbereich $50^{\circ}, 25$ Projektionen, Scandauer $>20$ s, Geometrie: gleichförmige Abtastung, Rekonstruktion: gefilterte Rückprojektion.

Die insgesamt bei 200 operierten Segmenten bei brusterhaltender Therapie angefertigten radiologischen Untersuchungen (digitale Vollfeldmammografie (Inspiration ${ }^{\mathrm{TM}}$ ) (2 Ebenen) (Standard); CMOS-Technologie (Bioptics) (2 Ebenen); Tomosynthese (1 Ebene) (25 Projektionen und Cine-Mode); wurden prospektiv 3 Radiologen mit unterschiedlicher Erfahrung in digitaler Mammografie zufallsverteilt (Zufallsgenerator), nur in Kenntnis des Ausgangsbefundes (histologisch Diagnose B5, diagnostisch BI-RADS 6), intraoperativ auf einem Befundungsmonitor präsentiert ( Abb. 1 bis 3). Die Ergebnisse der Befundung (Herd in allen 3 Ebenen mit ausreichendem Sicherheitssaum $(1,0 \mathrm{~cm})$ in der 


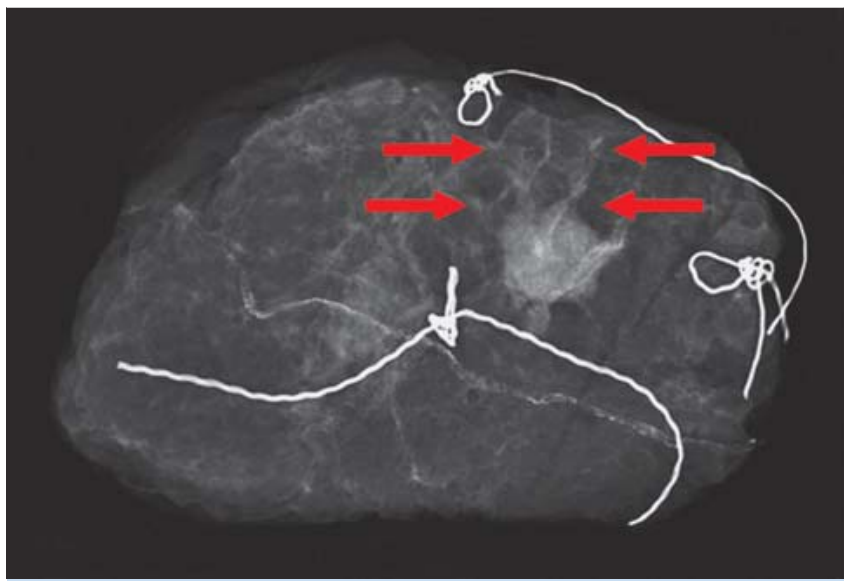

Fig. 1 Radiological imaging: Inspiration ${ }^{\mathrm{TM}}$. Magnification $1.0 \times 1.0$; invasive breast cancer PT1CNOM0; diameter of the tumour: $1.6 \mathrm{~cm}$; extent and differentiation of spiculations (arrows) from surrounding tissue could not be assessed.

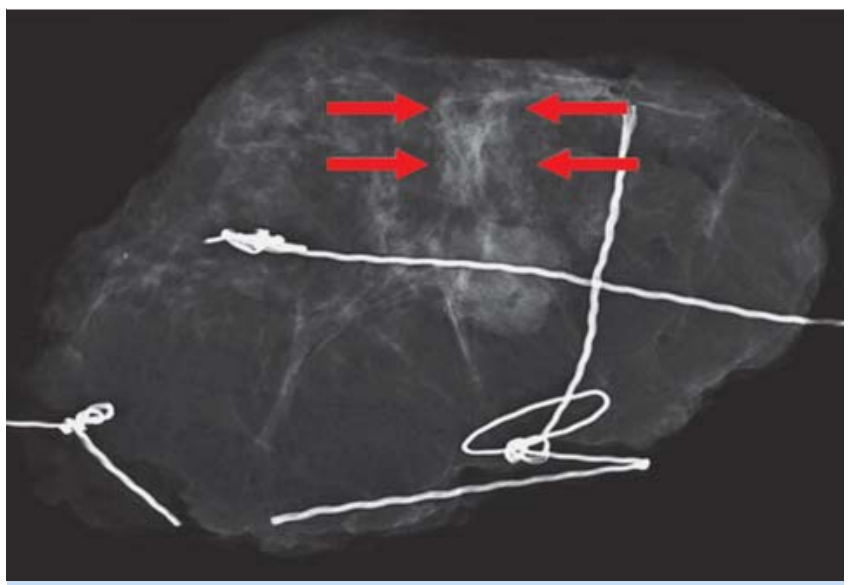

Fig. 2 BioVision ${ }^{\mathrm{TM}}$. Magnification $1.0 \times 1.0$; invasive breast cancer pT1CNOM0; diameter of the tumour: $1.6 \mathrm{~cm}$; extent and differentiation of spiculations (arrows) from surrounding tissue could not be assessed.

\section{Results}

\section{$\nabla$}

Out of a total of 200 patients with histologically confirmed breast cancer (BI-RADS 6), 156 patients required no further operative intervention (re-excision) after breast-conserving therapy - findings which were confirmed by radiographic imaging done directly (intraoperatively) during the primary intervention (focal lesion with sufficient safety margins $[1,0 \mathrm{~cm}]$ in all 3 types of radiological images). The radiogram in 2 planes, magnification 1.0:1.0 was defined as the standard ( $\bullet$ Fig. 1 ).

Subsequently, based on the standard radiographic image (Inspiration $^{\mathrm{TM}}, 2$ planes, magnification $\left.1.0: 1.0\right)$ and the pathological findings (10 specimens, no resection margins in healthy tissue; 34 specimens, lesion had a histological safety margin $<1.0 \mathrm{~cm}$ ), re-excision was carried out in 44 patients. Investigations carried out in parallel using CMOS technology (2 planes, magnification 1.0:1.0 (๑ Fig. 2) and Tomosynthesis (25 projections, cine mode, magnification $1.0: 1.0$ ) ( Fig. 3) showed that with Tomosynthesis only 28 patients would have required re-excision based on the above criteria.

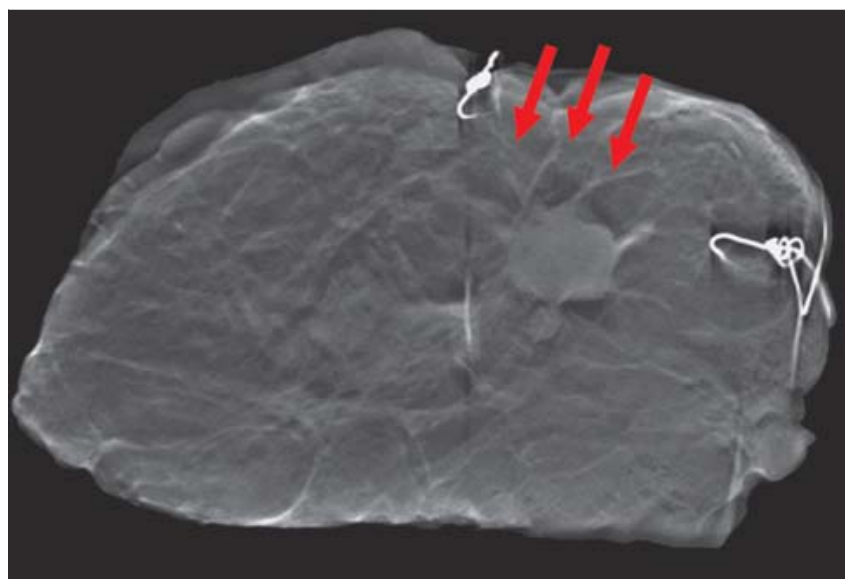

Fig. 3 Tomosynthesis, image 7 of 25 . Magnification $1.0 \times 1.0$; invasive breast cancer PT1cNOM0; diameter of the tumour: $1.6 \mathrm{~cm}$; extent and differentiation of spiculations (arrows) from surrounding tissue could be clearly evaluated (with imaging and then confirmed histologically).

Präparateradiografie (2 Ebenen bzw. 25 Projektionen) enthalten oder nicht) $[10,11]$ wurden mit dem abschließenden ausführlichen histologischen Gesamtbefund korreliert, wobei die 3 Befunder zu vergleichbaren Beurteilungen kamen.

\section{Ergebnisse \\ $\nabla$}

Bei den 200 Patientinnen mit histologisch gesichertem Mammakarzinom (BI-RADS 6) war bei 156 Patientinnen nach erfolgter brusterhaltender Therapie keine weitere operative Therapie (ReExzision) indiziert - bestätigt durch direkt während der primären Operation (intraoperativ) durchgeführte Präparateradiogramme (Herd in allen 3 Ebenen mit ausreichendem Sicherheitssaum $[1,0 \mathrm{~cm}]$ in der Präparateradiografie enthalten). Als Standard wurde dabei das Präparateradiogramm in 2 Ebenen, Vergrößerung 1,0:1,0 definiert ( $\bullet$ Abb. 1).

Anschließend wurde bei 44 Patientinnen aufgrund der Standardpräparateradiografie (Inspiration ${ }^{\mathrm{TM}}, 2$ Ebenen, Vergrößerung $1,0: 1,0)$ und des pathologischen Befundes (10 Präparate, Operation non in sano; 34 Präparate, Läsion histologisch mit einem Sicherheitsabstand $<1,0 \mathrm{~cm}$ ) eine Re-Exzision durchgeführt. Die parallel durchgeführte Untersuchung mit der CMOS-Technologie (2 Ebenen, Vergrößerung 1,0:1,0 ( $\odot$ Abb. 2) und der Tomosynthese (25 Projektionen und Cine-Mode, Vergrößerung 1,0:1,0) ( Abb. 3) ergab, dass bei der Tomosynthese lediglich bei 28 Pat. eine Re-Exzision aufgrund der angegebenen Kriterien notwendig sei.

Das endgültige histopathologische Ergebnis der 44 operierten Patientinnen (Re-Exzision) ergab dann das Ergebnis, dass bei $10 \mathrm{~Pa}-$ tientinnen die primäre Operation non in sano erfolgt war, aber nur bei 12 Patientinnen, dass der Sicherheitsabstand $<1,0 \mathrm{~cm}$ vorlag, d.h. keine Resttumoranteile mehr nachweisbar waren. Dies bedeutet, dass eine Re-Exzision aufgrund des endgültigen histopathologischen Befundes nur bei 22 Patientinnen gerechtfertigt gewesen wäre. Bei 22 Patientinnen konnte die Situation noch intraoperativ beim Ersteingriff geklärt werden, bei $22 \mathrm{~Pa}-$ tientinnen war ein 2-zeitiger Eingriff notwendig - es lagen bei 7 Patientinnen invasive bzw. bei 15 Patientinnen intraduktale Tumoranteile vor. 
Table 2 Sensitivity and specificity of all three systems (system 1: Inspiration ${ }^{\mathrm{TM}}$; system 2: BioVision ${ }^{\mathrm{TM}}$, and system 3: Tomosynthesis) as determined by 3 radiologists through the prospective assessment of 600 specimen radiograms/projection slides (cine mode) from 200 patients with a total of 200 malignant lesions.

\begin{tabular}{|lllllll} 
& Sensitivity (\%) & & \multicolumn{3}{c}{ Specificity (\%) } \\
Magnification & System 1 & System 2 & System 3 & System 1 & System 2 & System 3 \\
$1.0: 1.0$ & Inspiration & BioVision & Tomosynthesis & Inspiration & BioVision & Tomosynthesis \\
(all 3 systems) & $(85 \mu \mathrm{m})$ & $(50 \mu \mathrm{m})$ & $(85 \mu \mathrm{m})$ & $(85 \mu \mathrm{m})$ & $(50 \mu \mathrm{m})$ & $(85 \mu \mathrm{m})$ \\
\hline Radiologist 1 & 78.0 & 79.0 & 86.0 & 100.0 & 100.0 & 100.0 \\
\hline Radiologist 2 & 79.0 & 78.0 & 87.0 & 100.0 & 100.0 & 100.0 \\
\hline Radiologist 3 & 78.0 & 78.0 & 87.0 & 100.0 & 100.0 & 100.0 \\
\hline Mean & 78.3 & 78.3 & 86.6 & 100.0 & 100.0 & 100.0 \\
\hline
\end{tabular}

Table 3 Detailed representation of results (R0/R1) ( $n=200$ specimens) for the three diagnostic systems (system 1: Inspiration ${ }^{\mathrm{TM}}$; system 2: BioVision ${ }^{\mathrm{TM}}$; system 3: Tomosynthesis) and the final histological result.

\begin{tabular}{|c|c|c|c|}
\hline$n=200$ & & & \\
\hline Diagnostics: histology & $R 0: n=156$ & & \\
\hline 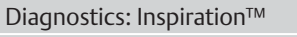 & $\mathrm{R} 1: \mathrm{n}=44$ & & \\
\hline Diagnostics: BioVision ${ }^{\top \mathrm{M}}$ & $R 1: n=44$ & Histology & $\begin{array}{l}R 1: n=22 \\
\text { R0: } n=22\end{array}$ \\
\hline Diagnostics: Tomosynthesis & $\mathrm{R} 1: \mathrm{n}=28$ & & \\
\hline
\end{tabular}

The final histopathological results for 44 operated patients (reexcision) showed that while in 10 patients the primary operation had not included the resection of margins in healthy tissue, only 12 other patients had a safety margin of $<1.0 \mathrm{~cm}$, i.e. with no tumour remnants. This means that re-excision based on the final histopathological results would have been only justified in $22 \mathrm{pa}-$ tients. In 22 patients, the situation could be settled intraoperatively during the primary intervention, while in 22 patients a second intervention was required. Seven of these patients had invasive and 15 patients had intraductal tumour lesions.

A retrospective analysis $(n=44)$ which directly compared the radiographic images demonstrated an increased sensitivity of Tomosynthesis of $8 \%(\mathrm{p}<0.05)$ compared to both the BioVision ${ }^{\mathrm{TM}}$ (CMOS technology) and the Inspiration ${ }^{\mathrm{TM}}$ at a magnification of $1.0: 1.0$, i.e., using Tomosynthesis re-excision would not have been necessary in 16 patients ( Tables 2 and $\mathbf{3}$ ).

\section{Discussion \\ $\nabla$}

The effectiveness of breast cancer therapy [1-7] can be increased during operations, i.e. intraoperatively, through the use of additional radiological imaging [10-11]. This should be done, where possible, on 2 planes in accordance with the specifications of the European Guidelines and the S3 Guidelines [8-11]. This would give surgeons immediate confirmation of the success of resection during an intervention. Direct imaging of the specimen over 2 planes could permit re-resection to be carried out immediately when the pathology has not been entirely contained by the specimen. The use of digital film combined with conventional analogue mammography already represents a big step forward. The procedure uses CCD chips and thus offers the possibility of using PACS, but its DQE (detector quantum efficiency) is much poorer compared to that of more recent fully digital systems [14-22]. In the last 10-15 years, developments in radiology have focussed mainly on the creation of specimen radiograms which can be viewed at many different locations (e.g. the operating room, the
Die retrospektive Analyse $(n=44)$ im direkten Vergleich der Präparateradiogramme ergab eine Steigerung der Sensitivität der Tomosynthese sowohl gegenüber des BioVision ${ }^{\mathrm{TM}}$ (CMOS-Technologie) als auch dem Inspiration ${ }^{\mathrm{TM}}$ bei einer Vergrößerung von $1,0: 1,0$ von $8 \%(\mathrm{p}<0,05)$, d. h. bei 16 Patientinnen wäre mit der Tomosynthese eine Re-Exzision nicht notwendig gewesen (- Tab. 2 und 3).

\section{Diskussion}

Die Effektivität in der Behandlung des Mammakarzinoms [1-7] kann während, d.h. intraoperativ, durch zusätzliche Präparateradiogramme gesteigert werden [10-11]. Diese sollten nach Vorgabe der European Guidelines und der S3-Leitlinien nach Möglichkeit in 2 Ebenen durchgeführt werden [8-11]. So besteht die Möglichkeit, noch während der Operation die unmittelbare Bestätigung für den Operationserfolg zu erhalten. Durch die direkte Darstellung des Präparats in 2 Ebenen kann bei nicht im Präparat enthaltener Pathologie unmittelbar eine Nachresektion erfolgen. Ein großer Fortschritt war sicher, dass über die Nutzung konventioneller, analoger Mammografieeinrichtungen die digitalen Speicherfolien zum Einsatz kamen. Dieses Verfahren beruht auf CCD-Chips und der damit verbundenen Möglichkeiten der Nutzung des PACS - hat aber zu den aktuell vorhandenen volldigitalen Systemen eine deutlich schlechtere DQE (Detector Quantum Efficiency) [14-22]. In den letzten 10-15 Jahren gab es nun Entwicklungen von Röntgeneinrichtungen ausschließlich zur Erstellung von Präparateradiogrammen, welche an unterschiedlichen Orten benutzt werden konnten (z.B. OP, Röntgenabteilung, Pathologie). Hierbei gab es ebenfalls eine Innovation von konventionellen, über Speicherfolien zu volldigitalen Systemen [14-22] bis hin zur CMOS-Technologie [23] und dem Einsatz der Tomosynthese [24]. Durch die Neuentwicklung der digitalen Brusttomosynthese (DBT) [25-32] besteht nun auch die Möglichkeit der Erstellung intraoperativer Präparateradiogramme [24]. Die 3 Untersucher beurteilten zufallsverteilt (Zufallsgenerator), nur in Kenntnis des Ausgangsbefundes (histologisch Diagnose B5, diagnostisch BI-RADS 6), intraoperativ auf einem Befundungsmonitor die 200 Präparate (Präparateradiografie) mit dem BioVision $^{\mathrm{TM}}$-System, 2 Ebenen (System 2) und der Tomosynthese (1 Ebene) einerseits sowohl anhand der Projektionsbilder als auch im Cine-Mode (System 3), wobei als Standard das Präparateradiogramm in 2 Ebenen (System 1) definiert wurde, aufgrund dessen bei 44 Patientinnen eine Re-Exzision durchgeführt wurde. Im direkten Vergleich der Ergebnisse der Präparateradiografie von Tomosynthese zu BioVision ${ }^{\mathrm{TM}}$ und Inspiration ${ }^{\mathrm{TM}}$ ergab sich eine Steigerung der Sensitivität von 8\% ( $p<0,05)$. Der Grund hierfür mag darin bestehen, dass die Tomosynthese es ermöglicht, 
radiology department, the pathology department). Here too, there has been a gradual switch from conventional systems using slides to fully digital systems [14-22] to CMOS technology [23] and Tomosynthesis [24]. The most recent developments in digital breast tomosynthesis (DBT) [25-32] make it possible to create specimen radiograms intraoperatively [24]. The 3 investigating radiologists assessed 200 specimens (specimen radiograms) randomly assigned to them (random generator) with only a prior knowledge of the initial pathology findings (histological classification B5, diagnostic classification BI-RADS 6). Assessment of images created with the BioVision ${ }^{\mathrm{TM}}$ system (system 2: 2 planes) and with Tomosynthesis (1 plane) using both projection slides and the cine mode (system 3 ) was done intraoperatively. However, specimen radiographs in 2 planes (as created by system 1) were defined as the standard, and re-excision was performed in 44 patients based on this standard.

A direct comparison of the images created by Tomosynthesis compared to those of the BioVision ${ }^{\mathrm{TM}}$ and the Inspiration ${ }^{\mathrm{TM}}$ showed an increase of sensitivity of $8 \%(p<0.05)$. The reason for this may be that Tomosynthesis makes it possible to characterise the structure of breast parenchyma in the mammogram ( $c-c$ and obliq.) more precisely [30]. The specimen radiograms showed a better differentiation (spiculations of the tumorous lesion and DCIS compared to surrounding benign parenchyma). The fact that the radiologists were aware of the initial diagnosis and findings constitutes a certain limitation of the results.

The breast re-excision rate after primary breast-conserving therapy (BET) for cancer lesions previously confirmed histologically (histological classification: B5 and therefore BI-RADS 6) was $22 \%$ in our historical collective of patients but also in the retrospective assessment of our current collective. In the literature, the breast re-excision rate after BET is reported to be between 10 and $57 \%$, depending on the definition of the safety margin $[12,13]$. Quality only improved substantially with the introduction of the S3 Guidelines, the treatment of patients in certified breast centres, and the introduction of digital technologies [12]. In future, to further reduce the re-excision rate, not simply in order to comply with the European Guidelines or S3 Guidelines but to spare patients from having to undergo a second operative intervention with all the side effects this entails, a volumetric assessment based on Tomosynthesis could offer an important contribution to mammography [31]. However, it should be noted that our Tomosynthesis results corresponded to or even exceeded those provided by digital specimen radiograms, even with the use of zooming [23,33-36]. To potentially improve results and thus the quality of specimen radiograms even more and to further reduce the rate of re-excisions, comparative studies of Tomosynthesis and specimen radiograms in real magnification (e.g. by a factor of 2.0) but without zooming would be necessary. The future use of 3-D technology in mammography including 3-D specimen radiograms also offers the potential to improve quality. The prerequisite for this is the integration of all components in an efficient PACS system for the online exchange of information between diagnosticians, pathologists and surgeons as part of a quality management drive [37]. die Textur des Brustparenchyms in der Übersichtsmammografie (c-c und obliq.) genauer zu charakterisieren [30] und dies sich nun in den Präparateradiogrammen ebenfalls zeigt: bessere Abgrenzbarkeit (Spiculae- und DCIS-Anteile des tumorösen Geschehens im Vergleich zum übrigen benignen Drüsenparenchym). Eine gewisse Einschränkung der Ergebnisse war sicher dadurch gegeben, dass den Befundern der Ausgangsbefund bekannt war. Die Mamma-Re-Exzisionsrate nach primär brusterhaltender Therapie (BET) bei zuvor histologisch nachgewiesenem Mammakarzinom (histologisch: B5 und damit BI-RADS 6) beträgt in unserem historischen Patientinnenkollektiv, aber auch aktuell in unserer retrospektiven Auswertung 22\%, welche in Abhängigkeit des definierten Sicherheitssaums in der Literatur zwischen 10 und 57\% liegt $[12,13]$. Erst durch die Einführung der S3-Leitlinien und der Behandlung der Patientinnen in zertifizierten Brustzentren und digitaler Techniken ist es gelungen, die Qualität entscheidend zu verbessern [12]. Um eine Senkung der Re-Exzisionsrate, nicht um die European Guidelines oder S3-Leitlinien zu erfüllen, sondern insbesondere den Patientinnen einen operativen Zweiteingriff mit all seinen Nebenwirkungen zu ersparen, zu erreichen, könnte die auf der Basis der Tomosynthese zukünftig bestehende Möglichkeit der Volumetrie in der Mammografietechnik einen wesentlichen Beitrag leisten [31]. Darüber hinaus entsprechen bzw. übertreffen unsere Ergebnisse denen für Präparateradiogramme in digitaler Technik auch unter Verwendung des Zoomings [23,33-36]. Um eventuell die Ergebnisse und damit die Qualität der Präparateradiografie noch zu verbessern und die Rate an Re-Exzisionen weiter zu reduzieren, wären vergleichende Untersuchungen von Tomosynthese und Präparateradiografien in realer Vergrößerung (z. B. Faktor 2,0), jedoch kein Zooming, notwendig. Ein weiteres Potenzial der Qualitätssteigerung könnte sich aber auch durch die zukünftige 3-D-Technologie in der Mammografie einschließlich der dann auch zu erwartenden 3-D-Präparateradiografie ergeben - Voraussetzung hier$\mathrm{zu}$ ist die Integration aller Komponenten in ein leistungsfähiges PACS zum Online-Austausch von Diagnostiker, Pathologen und Therapeuten im Rahmen des Qualitätsmanagements [37].

\section{Interessenkonflikt}

$\nabla$

Nein.

\section{Affiliations}

${ }^{1}$ Gynäkologische Radiologie, Radiologisches Institut des Universitätsklinikums Erlangen, Erlangen

2 Radiologie, Radiologie Roth, Roth

3 Universitätsfrauenklinik, Universitätsklinikum Erlangen, Erlangen

${ }^{4}$ Radiologisches Institut, Universitätsklinikum Erlangen, Erlangen

${ }^{5}$ Direktor der Frauenklinik, Universitätsklinikum Erlangen, Erlangen

${ }^{6}$ Direktor des Radiologischen Instituts, Universitätsklinikum Erlangen, Erlangen

\section{Conflict of Interests}

$\nabla$

None. 


\section{References}

1 Ruckhaberle E, Solbach C, Kaufmann M. Conference report on the 31st annual San Antonio breast cancer symposium, December 10-14, 2008 "Continuing change in diagnostics and therapy of breast cancers from molecular basics to tailored therapy". Geburtsh Frauenheilk 2009; 69: 202-211

2 Kaufmann M, Rody A. Breast cancer: reduced mortality by early detection and adjuvant therapy. Geburtsh Frauenheilk 2009; 69: 218-232

3 Katalinic A. Breast cancer: declining mortality despite its increasing incidence. Geburtsh Frauenheilk 2009; 69: 237-239

4 Beckmann MW, Blohmer JU, Costa SD et al. Zurich consensus: German expert opinion on the St. Gallen votes on 15 March 2009 (11th international conference at St. Gallen: primary therapy of early breast cancer). Geburtsh Frauenheilk 2009; 69: 377-383

5 Ruckhaberle E, Rody A, Kaufmann M. Report of the 32nd annual St. Antonio breast cancer symposium, December 10-13, 2009 "An international scientific symposium for interaction and exchange among basic scientists and clinicians in breast cancer". Geburtsh Frauenheilk 2010; 70: 177-183

6 Fasching PA, Fehm T, Janni W et al. Breast cancer therapy - a state of the art review. Geburtsh Frauenheilk 2010; 70: 875-886

7 Fasching PA, Ekici AB, Adamietz BR et al. Breast cancer risk - genes, environment and clinics. Geburtsh Frauenheilk 2011; 71: 1056-1066

8 Perry NM. Quality assurance in the diagnosis of breast disease. Eur J Cancer 2001; 37: 159-172

9 Wallis M, Tardivon A, Helbich T et al. Guidelines from the European Society of Breast Imaging for diagnostic interventional breast procedures. Eur Radiol 2007; 17: 581-588

10 Albert US, Hrsg. S3-Leitlinie Brustkrebs-Früherkennung in Deutschland. 1. Aktualisierung 2008. www.senologie.org 2008. München: W. Zuckschwerdt-Verlag; 2008

11 Kreienberg R, Kopp I, Albert US et al. S3-Leitlinie Diagnostik, Therapie und Nachsorge des Mammakarzinoms. 1. Aktualisierung. www.senologie.org 2008. München: W. Zuckschwerdt-Verlag; 2008

12 Bani MR, Lux MP, Heusinger $K$ et al. Factors correlating with reexcision after breast-conserving therapy. EJSO 2009; 35: 32-37

13 D'Orsi CJ. Management of the breast specimen. Radiology 1995; 194 : 297-302

14 Grunert J-H, Wiechmann U, Gmelin E. Kompressionsfreie Mammapräparateradiographie in 2 Ebenen mit Hilfe eines Radiographiecontainers. Fortschr Röntgenstr 2003; 175: 413-417

15 Kürzl R, Baltzer J, Lohe KJ. Intraoperative specimen radiography in mammographically suspected, non palpable breast lesions. Experiences with the Faxitron unit. Fortschr Med 1979; 97: 1688-1690

16 Kürzl R, Lohe KJ, Baltzer J et al. Radiography used during operations for biopsies of non-palpable lesions of the breast which have been suspected because of mammography. J Gynecol Obstet Biol Reprod 1981; 10: 431-434

17 Moritz JD, Westerhof JP, Funke $M$ et al. Ein neues Präparatradiographiegerät mit maximal 20facher Vergrößerung für die Mammadiagnostik. Fortschr Röntgenstr 1998; 168: 133-138

18 Grunert JH, Borchert B, Farber A et al. Mikrokalknachweis in MammaExzisionspräparaten mit Hilfe der DIMA-Vergrößerungspräparateradiographie vierfach im Vergleich zur konventionellen Vergrößerungsradiographie eineinhalbfach. Fortschr Röntgenstr 1999; 171: 302-306

19 Grunert J-H, Flemming P, Borchert B et al. Vergrößerungspräparateradiographie als Qualitätskontrolle der Mikrokalkerfassung im Rahmen der histopathologischen Aufarbeitung von Mammabiopsiegewebe. Fortschr Röntgenstr 2000; 172: 68-72
20 Diekmann F, Grebe S, Bick U et al. Digitale intraoperative Präparateradiographie bei diagnostischen Exzisionen nicht palpabler Läsionen der Brust. Fortschr Röntgenstr 2000; 172: 969-971

21 Muttalib M, Tisdall M, Scawn R et al. Intra-operative specimen analysis using faxitron microradiography for excision of mammographically suspicious, non-palpable breast lesions. The Breast 2004; 13: 307-315

22 Schulz-Wendtland R, Wenkel E, Wacker T et al. Quo vadis? Trends in digital mammography. Geburtsh Frauenheilk 2009; 69: 108-117

23 Schulz-Wendtland R, Bani MR, Lux MP et al. CMOS-Technologie für intraoperative digitale Präparateradiografien. Geburtsh Frauenheilk 2011; 71: 525-529

24 Schulz-Wendtland R, Dilbat G, Bani MR et al. Use of tomosynthesis in intraoperative digital specimen radiography - is a reduction of breast reexcision rates possible? Geburtsh Frauenheilk 2011; 71: 1080-1084

25 Niklason LT, Christian BT, Niklason LE et al. Digital tomosynthesis in breast imaging. Radiology 1997; 205: 399-406

26 Schulz-Wendtland $R$, Wenkel E, Lell $M$ et al. Experimental phantom lesion detectability study using a digital breast tomosynthesis prototype system. Röfo 2006; 178: 1219-1223

27 Poplack SP, Tosteson TD, Kogel CA et al. Digital breast tomosynthesis: initial experience in 98 women with abnormal digital screening mammography. AJR 2007; 189: 616-623

28 Good WF, Abrams GS, Catullo VJ et al. Digital breast tomosynthesis: a pilot observer study. AJR 2008; 190: 865-869

29 Gur D, Abrams GS, Chough DM et al. Digital breast tomosynthesis: observer performance study. AJR 2009; 193: 586-591

30 Kontos D, Bakic PR, Carton AK et al. Parenchymal texture analysis in digital breast tomosynthesis for breast cancer risk estimation: a preliminary study. Acad Radiol 2009; 16: 283-298

31 Schulz-Wendtland R, Fuchsjäger M, Wacker T et al. Digital mammography: an update. Eur J Radiol 2009; 72: 258-265

32 Teertstra HJ, Loo CE, van den Bosch MA et al. Breast tomosynthesis in clinical practice: initial results. Eur Radiol 2010; 20: 16-24

33 Schulz-Wendtland R, Hermann K-P, Wenkel E et al. Vergleich der klinischen Wertigkeit von Zielaufnahmen mit reduzierter Pixelgröße des Detektors und digitalem Monitorzooming bei der Beurteilung von Mikrokalzifikationen in der digitalen Mammographie. Fortschr Röntgenstr 2007; 179: 492-496

34 Haygood TM, Arribas E, Brennan PC et al. Conspicuity of microcalcifications on digital screening mammograms using varying degrees of monitor zooming. Acad Radiol 2009; 16: 1509-1517

$35 \mathrm{Kim}$ MJ, Youk JH, Kang DR et al. Zooming method (x 2.0) of digital mammography vs. digital magnification view (x 1.8) in full-field digital mammography for the diagnosis of microcalcifications. Br J Radiol 2010; 83: 486-492

36 Koutalonis M, Delis H, Pascoal A et al. Can electronic zoom replace magnification in mammography? BJR 2010; 83: 569-577

37 Schulz-Wendtland R, Bani MR, Lux MP et al. Pilot study for the detection of simulated lesions at a 2D resp. 3D digital full-field mammography system with a new developed high resolution detector on the base of two shifts of a-Se. Geburtsh Frauenheilk 2012; 72: 408-411

Deutschsprachige Zusatzinformationen online abrufbar unter: www.thieme-connect.de/ejournals/toc/gebfra. 